\title{
The Correlation of SCL-90-R Anxiety, Depression, Somatization Subscale Scores with Chronic Low Back Pain
}

\author{
Utku ADILAY ${ }^{1}$, Bulent GUCLU², Murat GOKSEL ${ }^{3}$, Semih KESKIL ${ }^{4}$ \\ ${ }^{1}$ Balikesir University, School of Medicine, Department of Neurosurgery, Balikesir, Turkey \\ ${ }^{2}$ Dr. Lutfi Kirdar Kartal Training and Research Hospital, Neurosurgery Clinic, Istanbul, Turkey \\ ${ }^{3}$ Bursa Yuksek Ihtisas Training and Research Hospital, Neurosurgery Clinic, Bursa, Turkey \\ ${ }^{4}$ Yüksek Ihtisas University, Ankara, Turkey
}

\section{ABSTRACT}

AIM: To investigate the correlation of the Symptom Checklist-90-R (SCL-90-R) anxiety, depression, and somatization subscale scores with chronic low back pain (LBP).

MATERIAL and METHODS: Seventy-five patients who were admitted with the complaint of chronic LBP (patient group) and 75 healthy persons (control group) were evaluated. SCL-90-R anxiety, depression, and somatization subscale scores of patients having chronic LBP and healthy persons were measured. The mean values were paired and using two tailed test they were statistically evaluated.

RESULTS: The difference between SCL-90-R anxiety subscale subscores of patients having chronic LBP and healthy persons was not statistically significant $(p>0.05$ ). The difference between SCL-90-R depression subscale subscores of patients having chronic LBP and healthy persons was not statistically significant $(p>0.05)$. The difference between SCL-90-R somatization subscale subscores of patients having chronic LBP and healthy persons was statistically significant $(p<0.05)$.

CONCLUSION: SCL-90-R somatization subscale subscores are higher in patients with low back pain. The treatment of low back pain can be more successful when combined with the treatment of somatization.

KEYWORDS: Anxiety, Depression, Low back pain, SCL-90-R, Somatization

\section{INTRODUCTION}

L ow back pain (LBP) is one of the most common complaints in general medical practice and affects nearly $80 \%$ of the general population (18). This symptom is the second most common cause of hospitalization in the working age group after the common cold. Every human being in the society has at least one attack of low back pain during his/her lifetime. Cases may come with an acute history or as a chronic complaint. LBP is a complex condition produced by multiple factors (Table I). Psychosocial difficulties and psychological factors might be associated with LBP (12). Despite the recognition that excessive pain complaints are associated with psychological problems, the exact relationship between pain behavior and psychological factors are not very clear. Besides the importance of selecting patients with "psychogenic diseases" as stated in Table I among other cases, the anxiety, depression, somatization, caused by pain can provoke pain itself although correct therapy is used especially in chronic cases.

Bener et al. reported that depression and somatization were very common in LBP patients (2). Also, in another study, they observed a high rate of co-morbidity of somatization, depression, anxiety, and stress in the studied population and indicated a strong association between these psychological 
disorders in patients (3). In a recent study, they stated that psychological distress such as anxiety, depression, and somatization were more prevalent in LBP patients compared to patients without LBP (4).

SCL-90- $R$ is rarely used in psychiatry but it is commonly helpful in other medical disciplines or investigations $(5,8,10,11,13,14)$. The aim of this study was to investigate the correlation of the SCL-90-R anxiety, depression, and somatization subscale scores with chronic low back pain.

\section{MATERIAL and METHODS}

This cross-sectional retrospective study was conducted at the Neurosurgery Clinic of the Dr. Lutfu Kirdar Kartal Research and Training Hospital, and the Neurosurgery Clinic of the Yuksek Ihtisas Research and Training Hospital, between May 2013 and January 2015. Patients with chronic LBP who were hospitalised and evaluated radiologically and clinically were included in this study. Patients with spinal pathologies requiring surgery such as lumbar disc herniation, lumbar spondylolisthesis, lumbar spinal stenosis, who had the history of spinal surgery, who had LBP with less than 12 weeks pain history, who had other severe diseases that might cause anxiety, depression and somatization were excluded from study. Only 75 patients fulfilling these criteria, who had answered the SCL-90-R test, and who were between 18 and
65 years were found retrospectively. However, the control patients were not hospitalised. The sociodemographic data of this study population is given in Table II. SCL-90-R anxiety, depression and somatization subscale scores of patients having LBP and healthy people were measured and compared.

The Symptom Checklist- 90 was first introduced by Derogatis and Lipman, and it is now used in its revised form (SCL-90-R) by its authors (8). SCL-90-R is a relatively brief self-report psychometric instrument (questionnaire). It is composed of 90 questions and each item has 5 answer choices using five levels (between 0-4). The Likert-type question-answer model was used in SCL-90-R) (15) (none=0, too much=4). The questions are aimed to assess different symptoms and states of the mind. The diseases that are assessed by these question groups are somatization, obsessive-compulsive disease, inter-personal sensibility, depression, anxiety, hostility, phobic anxiety, paranoid thoughts, psychotic and additional symptoms. In this study, the SCL-90-R anxiety, depression, and somatization subscale scores were studied $(7,13)$.

All patients were administered the questionnaire after the clinical assessment, and after obtaining informed consent as explained by the doctor. Qualified nurses were trained to give information about the questionnaire to the patients. The patients were allowed to complete the questionnaire by themselves without any time restriction. The control group

Table I: Causes of Low Back Pain

\begin{tabular}{ccc}
\hline Mechanical Low Back Pain & Non-Mechanical Spinal Conditions & Other Diseases \\
\hline Herniated discs & Neoplasia & Pyelonephritis \\
\hline Degenerative processes & Multiple Myeloma & Prostatitis \\
\hline Lumbar strain, sprain & Lymphoma and leukemia & Endometriosis \\
\hline Spinal stenosis & Metastatic carcinoma & Renal disease \\
\hline Osteoporotic compression fracture & Spinal cord tumors & Nephrolithiasis \\
\hline Spondylolisthesis & Retroperitoneal tumors & Disease of pelvic organ \\
\hline Traumatic fracture & Primary vertebral tumors & Perinephric abscess \\
\hline Congenital disorders & Infection & Vascular disease \\
\hline Severe kyphosis & Osteomyelitis & Aortic aneurysm \\
\hline Severe scoliosis & Septic discitis & Gastrointestinal disease \\
\hline Transitional vertebrae & Paraspinous abscess & Pancreatitis \\
\hline Internal disc disruption & Epidural abscess & Penetrating ulcer \\
\hline Spondylolysis & Shingles & Psychogenic pain \\
\hline & Paget's disease of bone & \\
\hline & Inflammatory arthritis & \\
\hline & Psoriatic spondylitis & \\
\hline & Reactive arthritis & \\
\hline & Scheuermann's disease & \\
\hline
\end{tabular}


consisted of persons who declared that they had no acute or chronic disease.

All of the completed questionnaires were stored digitally after being controlled for possible mistakes. For statistical analysis, GraphPad Prism 5 for Windows (GraphPad Software Inc.) was used and the level for statistical significance was chosen as $p<0.05$.

\section{RESULTS}

For LBP patients with anxiety, the most frequent symptoms were "nervousness or shakiness inside" (77\%), "feeling tense or keyed up" (69\%), and "heart pounding or racing" (41\%) respectively. For LBP patients with depression, the most frequent symptoms were "feeling low in energy or slowed down" (64\%), "crying easily" (60\%), and "worrying too much about things" (57\%), respectively. For LBP patients with somatization, the most frequent symptoms were "pains in lower back" (100\%), "soreness of muscles" (87\%)", and "headaches" (41\%), respectively.

Anxiety, one of the subgroups of symptoms, was assessed by the group of questions which were paired (Figure 1) and looked for statistically significant difference between the groups using two tailed t test. The $p$ value was 0.1297 , showing the difference to be statistically non-significant.

Depression, another of the subgroups of symptoms, was assessed by the group of questions which were paired (Figure 2) and looked for statistically significant difference between the groups using two tailed t test. The $p$ value was 0.3006 , showing the difference to be statistically non-significant.

Somatization, the last one of the subgroups of symptoms, was assessed by the group of questions which were paired (Figure 3) and looked for statistically significant difference between the groups using two tailed t test. The $p$ value was 0.0228 $(<0.05)$, showing the difference to be statistically significant.

\section{DISCUSSION}

LBP is widely regarded as a biopsychosocial problem (17). Physical and psychological stressors are known to increase LBP risk. The aim of our study was to investigate the correlation between anxiety, depression, and somatization and LBP. We therefore used SCL-90-R as the questionnaire. SCL-90-R is a test that can be easily used in clinical practice individually and for groups without any need of help. The answers to the each question as none/too light/moderate/too much/highest degree were changed into numerical values as 0 to 4 .

When both organic and psychological health is impaired in any case, the anxiety is always associated with this degradation; in other words, the development of anxiety is expected (10). However, we found that patients with low back pain do not have accompanying statistically significant anxiety. This result is contradictory to the general medical concept that in every situation where health is disrupted, there is accompanying anxiety. In a study focused on the impact of anxiety or somatization on the occurrence of LBP, no association was

Table II: Sociodemographic Characteristics of the Study Population according to Low Back Pain

Low back pain $(n=75) \quad$ Control $(n=75) \quad$ Total $(n=150)$

\begin{tabular}{lccc}
\hline $\begin{array}{l}\text { Age (years) } \\
\text { Mean } \pm \text { SD }\end{array}$ & $46.45 \pm 13.35$ & $48.33 \pm 10.87$ & $47.39 \pm 11.95$ \\
\hline Sex, $\mathbf{n}(\%)$ & $43(58 \%)$ & $39(52 \%)$ & $82(55 \%)$ \\
$\quad$ Female & $32(42 \%)$ & $36(48 \%)$ & $68(45 \%)$ \\
$\quad$ Male & & & \\
\hline Marital status, $\mathbf{n}(\%)$ & $14(19 \%)$ & $16(21 \%)$ & $30(20 \%)$ \\
$\quad$ Single & $45(60 \%)$ & $43(57 \%)$ & $88(59 \%)$ \\
$\quad$ Married & $9(12 \%)$ & $11(15 \%)$ & $20(13 \%)$ \\
$\quad$ Divorced & $7(9 \%)$ & $5(7 \%)$ & $12(8 \%)$ \\
$\quad$ Widowed & & & \\
\hline Employement status, $\mathbf{n}(\%)$ & $36(48 \%)$ & $15(52 \%)$ & $29(50 \%)$ \\
$\quad$ Currently working & $14(19 \%)$ & $21(28 \%)$ & $46(31 \%)$ \\
$\quad$ Unemployement & $25(33 \%)$ & $10(13 \%)$ & $21(14 \%)$ \\
$\quad$ Housewife & $11(15 \%)$ & $15(20 \%)$ & $29(20 \%)$ \\
\hline Educational level attained & $14(19 \%)$ & $20(27 \%)$ & $42(28 \%)$ \\
$\quad$ No education & $22(29 \%)$ & $22(29 \%)$ & $41(27 \%)$ \\
$\quad$ Primary school & $19(25 \%)$ & & $17(11 \%)$ \\
$\quad$ Junior secondary school & $9(12 \%)$ & & \\
$\quad$ Senior secondary school & & & \\
$\quad$ Tertiary & & & \\
\hline
\end{tabular}


found with anxiety (6). However in another study, anxiety was more prevalent in LBP patients compared to patients without LBP (4). According to our study LBP patients do not have more anxiety than healthy patients. The help of anti-anxiolytic therapy for the treatment of LBP is the subject of another study.

Depression, is a frequent a companion of chronic pain. Depression is also the most common type of mental disorder. Depression resulting from chronic pain can become consuming, and have the potential to significantly worsen and prolong the pain. Increased pain can, in turn, lead to increased depression, creating a cycle of depression and pain that can be difficult to break (16). Our findings did not show a statistically significant difference for depression subscale scores between the patient group and the control group. As a result of the evaluation of the depression subscale of the SCL-90-R, depression does not have any clinical and scientific importance

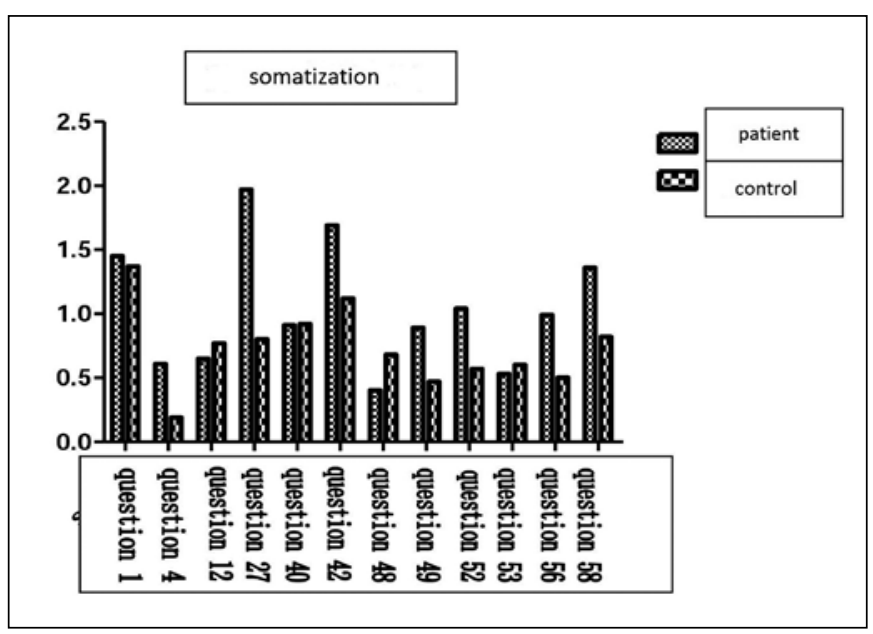

Figure 1: The questions for the SCL-90R anxiety subgroup.

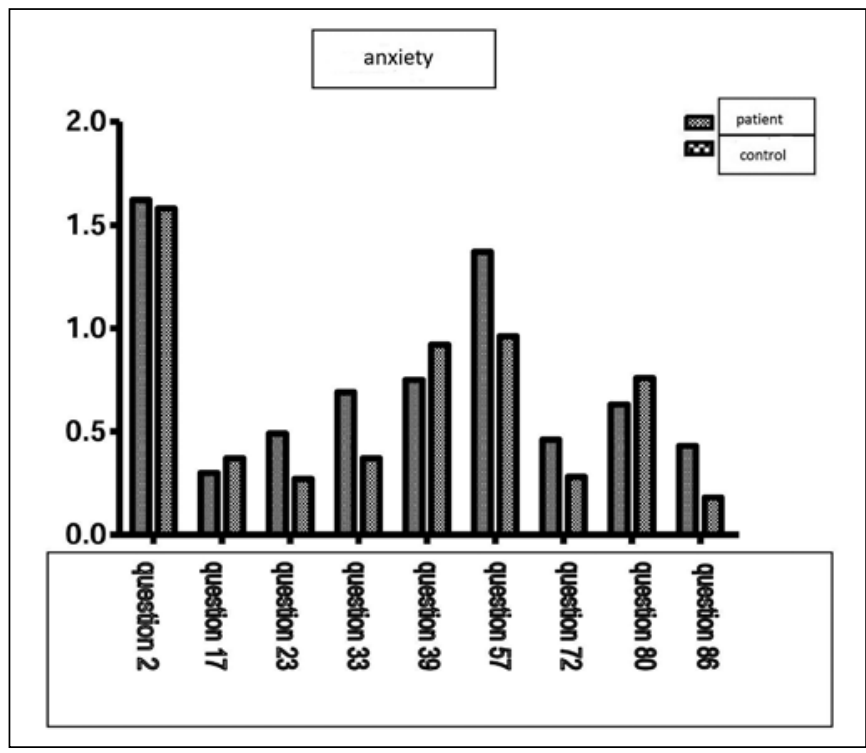

Figure 2: The questions for the SCL-90R depression subgroup. and interest in clinical assessment of back pain. However this is contradictory to general practice of the doctors dealing with LBP. Most doctors dealing with LBP prescribe anti-depressant drugs to patients with LBP. Similar to our results, Dickens et al. showed that patients with and without excessive pain behavior had similar levels of anxiety and depression, especially in women (9). However Beneret al. stated that depression was more prevalent in LBP patients compared to patients without LBP. The correlation between depression and LBP can be evaluated with new studies. And also the effectiveness of anti-depressant drugs for the treatment of LBP is the subject of another study.

When the somatization subscale scores SCL-90-R were evaluated, somatization measurement showed a statistically significant difference between patients and control groups. Somatization can be defined as expression with somatic complaints of a spiritual problem. This result can be interpreted as, among individuals admitted to our clinic with complaint of chronic LBP, the mechanism of this symptom is in the form of a somatization mechanism. Evaluation of our patients with higher scores on somatization, as whether how many of them also had anatomical and mechanical disorders, was not made. The correlation between higher somatization scores and anatomical and mechanical disorders of patients having LBP should be evaluated in other studies. Our findings for the high correlation between somatization and LBP are similar to other studies $(4,6)$. Treatment of somatization should be added to the treatment of patients with LBP. And also the effectiveness of the treatment of the somatization for the treatment of LBP is the subject of another study.

Studies that have measured the sensitivity and reliability of the SCL-90-R reached a common conclusion that the overall result of this test reliably showed the presence of psychopathology in general subjects. However, for the subscales, the overall outcome of the test is not reliable and sensitive. It is possible to argue that the results of this study are supporting this

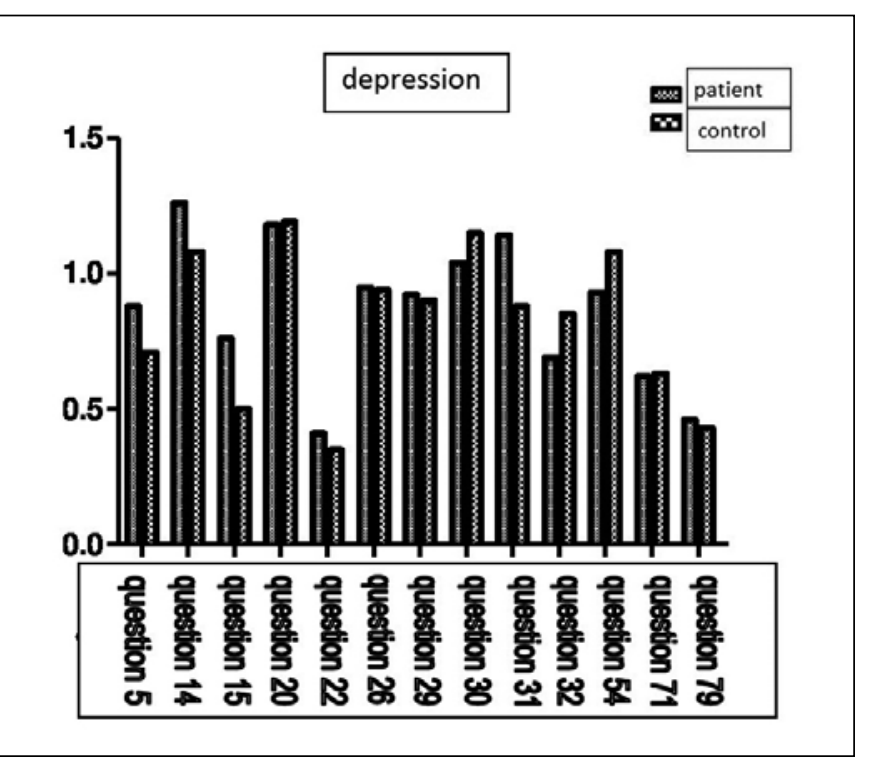

Figure 3: The questions for the SCL-90R somatization subgroup. 
general belief. A recent study also showed that a poor surgical outcome, especially resistant pain despite a postoperative radiological examination that conforms to expectations, is mainly associated with high scores on the SCL-90-R scale. These patients commonly feel that the operation "did not work", have worse interpersonal relations with the hospital staff, suffer from depression and conversion, demonstrate resistance to walking or sitting due to the fear of disturbing the operative site, have fear of feeling "still ill" and show an increased tendency to using pain killers (1).

Diagnosis and differential diagnosis of patients with LBP is considered by more than one medical discipline, and a process that requires active participation of the science of nursing. Looking at this process in terms of nursing, the necessity of putting forward correct nursing diagnoses in these cases is apparent. Correct nursing diagnosis is the foundation of the correct nursing approach. Some nursing diagnoses for patients with LBP include such titles as anxiety, acute pain, chronic pain, severe pain and impaired physical movement. Especially in patients with chronic pain, inability to cope with the diagnosis also can be added to these diagnoses. This study investigated the correlation of the SCL-90-R anxiety, depression, and somatization subscale scores with LBP.

The current approach to patients with LBP is a multi-faceted approach that requires team work and is considered as a multifaceted medical process. We think that the SCL90-R as added to the diagnosis and treatment of patients with LBP would help nurses and physicians reach a more accurate, complete and reliable conclusion. The relation of anxiety, depression, and somatization with LBP, and whether anxiety, depression, and somatization cause LBP even after the treatment of anatomical and mechanical disorders of the back causing pain, are very important. The doctors may treat anatomical and mechanical disorders of the back, but patients may have LBP even after this treatment. This may be due to anxiety, depression, and somatization of the patient. We thought that anxiety, depression, and somatization might cause LBP after the treatment of anatomical and mechanical disorders of the back. However, we only found a statistically significant correlation between somatization and LBP. We can only argue that somatization treatment should be added to the treatment of LBP. The correlation between somatization and $\mathrm{LBP}$, and the role of the somatization treatment in the treatment of LBP are the subject to larger studies. The result of these studies may add new treatment protocols to the treatment of LBP. Although we did not find statistically significantly higher scores for anxiety and depression in patients with LBP, we still argue that anxiety and depression can be related with LBP. The treatment of anxiety and depression in these patients may help the improvement of LBP. More complex studies are needed to reach more precise conclusions.

\section{- CONCLUSION}

Our study showed that SCL-90-R somatization subscale scores are higher in patients with LBP. The treatment of LBP may be more successful when combined with the treatment of somatization.

\section{REFERENCES}

1. Bademci G, Aslan N, Yucel E, Saygun M, Ozen NE, Ulu $\mathrm{N}$, Evliyaoglu C, Keskil S: Correlation of psychological assessment using SCL-90-R scale with surgical outcome. Turk Neurosurg 15:1-3, 2005

2. Bener A, El-Rufaie OF, Kamran S, Georgievski AB, Farooq A, Rysavy M: Disability, depression and somatization in low back pain population. Int J Rheum Dis 9(3): 257-263, 2006

3. Bener A, Al-Kazaz M, Ftouni D, Al-Harthy M, Dafeeah EE: Diagnostic overlap of depressive, anxiety, stress, and somatoform disorders in primary care. Asia Pac Psychiatry 5(1): E29-38, 2013

4. Bener A, Verjee M, Dafeeah EE, Falah O, Al-Juhaishi T, Schlogl J, Sedeeq A, Khan S: Psychological factors: Anxiety, depression, and somatization symptoms in low back pain patients. J Pain Res 6: 95-101, 2013

5. Bijur PE, Latimer CT, Gallagher EJ: Validation of a verbally administered numerical rating scale of acute pain for use in the emergency department. Acad Emerg Med 10: 390-392, 2003

6. Burton AK, McClune TD, Clarke RD, Main CJ: Long-term follow-up of patients with low back pain attending for manipulative care: Outcomes and predictors. Man Ther 9(1): 30-35, 2004

7. Cunha I, Brissos S, Dinis M, Mendes I, Nobre A, Passao V: Comparison between the results of the Symptom Check List 90-R of two different populations with temporal lobe epilepsy. Epilepsy Behav 4: 733-739, 2003

8. Derogatis LR, Lipman RS: The SCL 90: An outpatient psychiatric rating scale. Psychopharmacol Bull 9: 13-28, 1973

9. Dickens C, Jayson M, Creed F: Psychological correlates of pain behavior in patients with chronic low back pain. Psychosomatics 43(1): 42-48, 2002

10. Essau CA: Comorbidity of anxiety disorders in adolescents. Depres Anxiety 18: 1-6, 2003

11. Lynn SG, Bauch CD, Williams DE, Beatty CW, Mellon MW, Weaver AL: Psychologic profile of tinnitus patients using the SCL-90-R at tinnitus handicap inventory. Oto Neurotol 24: 878-881, 2003

12. Maniadakis N, Gray A: The economic burden of back pain in the UK. Pain 84(1): 95-103, 2000

13. Numan $\mathrm{N}$ : Exploration of adverse psychological symptoms in Yemeni khat users by the Symptoms Check List-90. Addiction 99: 61-65, 2004

14. Peveler RC, Fairburn CG: Measurement of neurotic symptoms by self-report questionnaire: Validity of the SCL-90-R. Psychol Med 20: 873-879, 1990

15. Reish T, Vijayananda S, Gekle W, Tschacher W: A qualitative follow-up assessment two years after inpatient crisis intervention. Psychiatr Prax 31: 16-22, 2004

16. Von Kofrr M, Simon G: The relationship between pain and depression. Br J Psychiatry Suppl 30:101-108, 1996

17. Waddell G: The back pain revolution. 2nd ed. London: Churchill Livingstone, 2004

18. Wedderkopp N, Leboeuf-Yde C, Anderson LB, Froberg $\mathrm{K}$, Hansen HS: Back pain reporting pattern in a Danish population-based sample of children and adolescents. Spine (Phila Pa 1976) 26(17): 1879-1883, 2001 